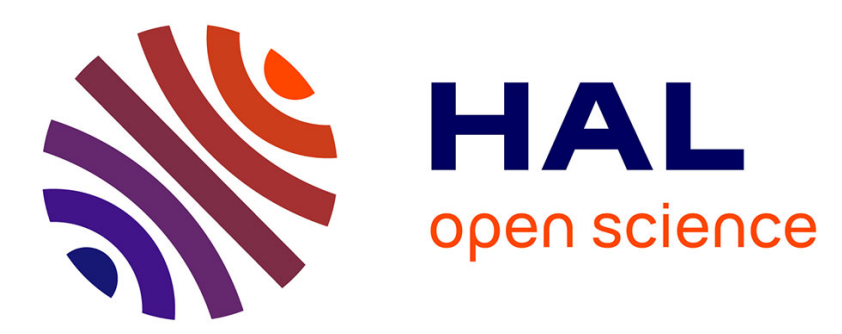

\title{
A New Approximate Godunov Scheme with Application to Dense Gas-Solid Flows
}

Thierry Gallouët, Jean-Marc Hérard

\section{To cite this version:}

Thierry Gallouët, Jean-Marc Hérard. A New Approximate Godunov Scheme with Application to Dense Gas-Solid Flows. 17th AIAA Computational Fluid Dynamics Conference,, Jun 2005, Toronto, Canada. 10.2514/6.2005-4860 . hal-01583851

\section{HAL Id: hal-01583851 \\ https://hal.science/hal-01583851}

Submitted on 3 Apr 2018

HAL is a multi-disciplinary open access archive for the deposit and dissemination of scientific research documents, whether they are published or not. The documents may come from teaching and research institutions in France or abroad, or from public or private research centers.
L'archive ouverte pluridisciplinaire HAL, est destinée au dépôt et à la diffusion de documents scientifiques de niveau recherche, publiés ou non, émanant des établissements d'enseignement et de recherche français ou étrangers, des laboratoires publics ou privés. 


\title{
A New Approximate Godunov Scheme With Application To Dense Gas-Solid Flows
}

\author{
Thierry Gallouet* \\ Université Aix-Marseille I, 13453 Marseille cedex 13, France \\ Jean-Marc Hérard ${ }^{\dagger}$ \\ EDF-DRD, 78401 Chatou cedex, France
}

\begin{abstract}
We present in this paper a new approximate Godunov solver called WFRoe which aims at enforcing the stability of the approximate Riemann solver VFRoe-ncv. In order to assess the method, we consider extreme situations and thus focus on the computation of some two-phase models which enable to account for flows highly loaded with particles. We show that the new solver WFRoe enables the computation of the propagation of shock waves over near-vacuum, and computes admissible states at each cell interface. Numerical tests confirm the efficiency of the scheme.
\end{abstract}

\section{Introduction}

One of the main problems when computing dense gas solid flows is that one needs to preserve the maximum principle for the void fraction (which is noted $\alpha$ herein) from a discrete point of view, and not only at the continuous level. This means that $\alpha(x, t)$ is expected to lie in $\left[0, \alpha_{\max }\right]$, where $\alpha_{\max } \leq 1$. This point is fundamental, otherwise, extensive use of the clipping approximation may result in many cases in a loss of conservation of mass of the mixture, or of particles, depending on the model retained. This is a well known fact for workers in the field of two phase flows, but also in classical single phase CFD (for shallow-water applications for instance).

Thus our goal here is to provide some way to handle computations from a practical point of view. When focusing on models for which the maximum principle holds in the solution of the exact Riemann problem, a straightforward and efficient idea simply consists in implementing the exact Godunov scheme. ${ }^{1}$ Focusing on the simple model A below for instance, one may benefit from the fact that the computed void fraction in each cell at the end of the time step simply represents the projection of the exact solution of two half Riemann problems, which guarantees that the maximum principle is fulfilled. This is due to the fact that the one dimensional scheme is consistant with the integral form of the conservation law see. ${ }^{2}$ Nonetheless, it is also well known that the Godunov scheme also suffers from two drawbacks. First of all, it is rather expensive, and this is even actually true in our case where the variations of functions involved in the solution of the Riemann problem will be hudge in the vicinity of an upper bound called $\alpha_{\max }$. Moreover, it is not so easy to implement, since one also needs to handle in an efficient way the vacuum areas. Another point is that the stability of strong double shock waves sometimes requires using rather small CFL number (that is CFL or order 0.1 instead of classical value 0.5$)$. All these restrictions lead us to investigate a new class of

*Professor, CMI-LATP, 39 rue Joliot Curie

${ }^{\dagger}$ Research Engineer, Département MFTT, 6 quai Watier, and Associate Research Director, CNRS, LATP, AIAA Member 
approximate Godunov schemes, which are quite different from the modified version of Roe scheme described in, ${ }^{3}$ and also differ from other recent proposals of Liou and coworkers $\left(\mathrm{see}^{4}\right)$. It indeed takes its grounds on the earlier approximate Godunov scheme called VFRoe-ncv.

The paper will be organised as follows. Section 2 will be devoted to the presentation of the approximate Godunov scheme introduced in, ${ }^{5}$ and to a new version called WFRoe, which aims at enforcing the numerical stability in some extreme conditions which are detailed in the following sections.

In the next section, main emphasis will be given on one model which describes the motion of clouds of particles in a vacuum of gas. We then will turn to a second model in section 4 which enables to describe a two-phase flow of gas loaded with round rigid smooth particles. This second model is a conservative hyperbolic model which inherits hyperbolicity, owing to the local equilibrium velocity assumption. In all cases, we prove that the maximum principle holds for the void fraction in the exact solution of the one dimensional Riemann problem.

In the remaining sections $5-6$, focus will be given on the numerical modelling of these two phase flow models. The main properties of schemes VFRoe-ncv and WFRoe are detailed in each case. It will be shown that the scheme WFRoe permits computation of the propagation of shock waves over near-vacuum, and computes admissible states at each cell interface.

\section{VFRoe-ncv and WFRoe schemes}

We recall herein the basis of VFRoe-ncv scheme, ${ }^{5}$ which has also been investigated and compared with various Riemann solvers in references, ${ }^{6},{ }^{7},{ }^{9} .8$ We restrict for the sake of simplicity to regular meshes of size $\Delta x$ such that: $\Delta x=x_{i+\frac{1}{2}}-x_{i-\frac{1}{2}}, i \in \mathbb{Z}$. The time step is $\Delta t=t^{n+1}-t^{n}, n \in \mathbb{N}$. We define $W: \mathbb{R} \times \mathbb{R}^{+} \rightarrow \mathbb{R}^{p}$ the exact solution of the hyperbolic system:

$$
\left\{\begin{array}{c}
\frac{\partial W}{\partial t}+\frac{\partial F(W)}{\partial x}=0 \\
W(x, 0)=W_{0}(x)
\end{array}\right.
$$

where the spatial flux $F$ is $C^{1}\left(\mathbb{R}^{p}, \mathbb{R}^{p}\right)$. Let $W_{i}^{n}$ be the approximate value of $\frac{1}{\Delta x} \int_{x_{i-\frac{1}{2}}}^{x_{i+\frac{1}{2}}} W\left(x, t^{n}\right) d x$. Integrating over $\left[x_{i-\frac{1}{2}} ; x_{i+\frac{1}{2}}\right] \times\left[t^{n} ; t^{n+1}\right]$ provides:

$$
W_{i}^{n+1}=W_{i}^{n}-\frac{\Delta t}{\Delta x}\left(\phi_{i+\frac{1}{2}}^{n}-\phi_{i-\frac{1}{2}}^{n}\right)
$$

where $\phi_{i+\frac{1}{2}}^{n}$ stands for the numerical flux through the interface $\left\{x_{i+\frac{1}{2}}\right\} \times\left[t^{n} ; t^{n+1}\right]$. The time step is in agreement with some CFL condition in order to gain stability. $\phi_{i+\frac{1}{2}}^{n}$ usually only depends on $W_{i}^{n}$ and $W_{i+1}^{n}$ when restricting to so-called first order schemes, that is $\phi_{i+\frac{1}{2}}^{n}=\phi\left(W_{i}^{n}, W_{i+1}^{n}\right)$. Whatever the scheme is, the numerical flux must comply with the consistent condition $\left(\mathrm{see}^{10}\right)$ :

$$
\phi(V, V)=F(V)
$$

We present now approximate Godunov fluxes $\phi\left(W_{L}, W_{R}\right)$ associated with the 1D Riemann problem:

$$
\left\{\begin{array}{l}
\frac{\partial W}{\partial t}+\frac{\partial F(W)}{\partial x}=0 \\
W(x, 0)= \begin{cases}W_{L} & \text { if } x<0 \\
W_{R} & \text { otherwise }\end{cases}
\end{array}\right.
$$

$$
2 \text { of } 14
$$


VFRoe-ncv scheme is an approximate Godunov scheme where the approximate value at the interface between two cells is computed as detailed below. Let us consider some change of variable $Y=Y(W)$ in such a way that $W_{, Y}(Y)$ is invertible. The counterpart of above system for regular solutions is:

$$
\frac{\partial Y}{\partial t}+B(Y) \frac{\partial Y}{\partial x}=0
$$

where $B(Y)=\left(W_{, Y}(Y)\right)^{-1} A(W(Y)) W_{, Y}(Y)(A(W)$ stands for the Jacobian matrix of flux $F(W))$.

Now, the numerical flux $\phi\left(W_{L}, W_{R}\right)$ is obtained by solving the linearized hyperbolic system:

$$
\left\{\begin{array}{l}
\frac{\partial Y}{\partial t}+B(\hat{Y}) \frac{\partial Y}{\partial x}=0 \\
Y(x, 0)= \begin{cases}Y_{L}=Y\left(W_{L}\right) & \text { if } x<0 \\
Y_{R}=Y\left(W_{R}\right) & \text { otherwise }\end{cases}
\end{array}\right.
$$

where $\hat{Y}$ agrees with the condition: $\hat{Y}\left(Y_{L}, Y_{L}\right)=Y_{L}$, and also $\hat{Y}\left(Y_{L}, Y_{R}\right)=\hat{Y}\left(Y_{R}, Y_{L}\right)$.

The classical choice for $\hat{Y}$ in $(2)$ is :

$$
\hat{Y}\left(Y_{L}, Y_{R}\right)=\hat{Y}^{(1)}\left(Y_{L}, Y_{R}\right)=\left(Y_{L}+Y_{R}\right) / 2
$$

Hence it enables to compute $Y^{*}\left(\frac{x}{t} ; Y_{L}, Y_{R}\right)$, and thus to set:

$$
\phi\left(W_{L}, W_{R}\right)=F\left(W\left(Y^{*}\left(0 ; Y_{L}, Y_{R}\right)\right)\right)
$$

In order to compute $Y^{*}\left(\frac{x}{t} ; Y_{L}, Y_{R}\right)$, we need to introduce $\widetilde{l_{k}}, \widetilde{\lambda_{k}}$ and $\widetilde{r_{k}}, k=1, \ldots, p$, left eigenvectors, eigenvalues and right eigenvectors of matrix $B(\hat{Y})$ respectively. The solution $Y^{*}\left(\frac{x}{t} ; Y_{L}, Y_{R}\right)$ of the linear Riemann problem (2) is defined everywhere (except along $\frac{x}{t}=\widetilde{\lambda_{k}}$ ):

$$
\begin{aligned}
Y^{*}\left(\frac{x}{t} ; Y_{L}, Y_{R}\right) & =Y_{L}+\sum_{\frac{x}{t}>\widetilde{\lambda_{k}}}\left({ }^{t} \widetilde{l_{k}} \cdot\left(Y_{R}-Y_{L}\right)\right) \widetilde{r_{k}} \\
& =Y_{R}-\sum_{\frac{x}{t}<\widetilde{\lambda_{k}}}\left({ }^{t} \widetilde{l_{k}} \cdot\left(Y_{R}-Y_{L}\right)\right) \widetilde{r_{k}}
\end{aligned}
$$

Combining the last equalities enables to write the latter in a slightly different form:

$$
Y_{R}-Y_{L}=\sum_{k=1, p}\left({ }^{t} \widetilde{l_{k}} \cdot\left(Y_{R}-Y_{L}\right)\right) \widetilde{r_{k}}=\sum_{k=1, p} \tilde{\alpha}_{k} \widetilde{r_{k}}
$$

setting: $\tilde{\alpha}_{k}={ }^{t} \tilde{l_{k}} \cdot\left(Y_{R}-Y_{L}\right)$.

The explicit form of the Finite Volume method VFRoe-ncv is:

$$
W_{i}^{n+1}-W_{i}^{n}+\frac{\Delta t}{\Delta x}\left(F\left(W\left(Y^{*}\left(0 ; Y_{i}^{n}, Y_{i+1}^{n}\right)\right)\right)-F\left(W\left(Y^{*}\left(0 ; Y_{i-1}^{n}, Y_{i}^{n}\right)\right)\right)\right)=0
$$

Nonetheless, when one focuses on the first time iteration of a shock tube problem for instance, it obviously occurs that the intermediate state computed by VFRoe-ncv with the choice $\hat{Y}\left(Y_{L}, Y_{R}\right)=\hat{Y}^{(1)}\left(Y_{L}, Y_{R}\right)$-using previous notations- is close to the intermediate state of the exact solution of the Riemann problem, but the numerical wave speeds are poor approximations of exact wave speeds. For instance, we consider isentropic Euler equations with perfect gas EOS. Using initial condition $U_{L}=U_{R}=0, P_{L}=10^{5}$ and $P_{R}=10^{4}$ (where 
$\mathrm{L}$ and $\mathrm{R}$ subscripts refer to left and right state for velocity $\mathrm{U}$ and pressure $\mathrm{P}$ ), the average will be based on $\hat{U}=0$ which is not a fair approximation of the exact value of the intermediate state $U_{1}$ which is close to $c_{1}$. This has at least two drawbacks. First of all, the flow may be locally supersonic, whereas predicted values of characteristics with the choice $\hat{Y}\left(Y_{L}, Y_{R}\right)=\hat{Y}^{(1)}\left(Y_{L}, Y_{R}\right)$ are subsonic, which may result in some unphysical upwinding, and behind in a loss of stability. This may also be emphasised by the fact that the time step will be twice the admissible value in this example (since the true value of $U_{1}+c_{1}$ is about twice $\hat{U}+\hat{c}$ ), which of course may also lead to a loss of stability . Actually, practical computations show that after a few (typically 5) iterations, the cell values computed by the Godunov scheme and the standard VFRoe-ncv scheme can hardly be distinguished. So this (sometimes) poor approximation of numerical wave speeds does not have a real influence on the whole computation, in this particular case.

Moreover, the problem may even become worse in some situations involving at least two strong shock waves, as will be seen afterwards. By the way, it seems worth recalling that the (exact) Godunov scheme may also encounter some difficulties in this situation.

Thus, in order to enforce the stability of the approximate Godunov scheme VFRoe-ncv, we propose now to set :

$$
\hat{Y}\left(Y_{L}, Y_{R}\right)=\hat{Y}^{(2)}\left(Y_{L}, Y_{R}\right)=Y^{*}\left(0 ; Y_{L}, Y_{R}\right)
$$

where $Y^{*}\left(0 ; Y_{L}, Y_{R}\right)$ issues from a first computation with $\hat{Y}^{(1)}\left(Y_{L}, Y_{R}\right)=\left(Y_{L}+Y_{R}\right) / 2$. This new value $\hat{Y}^{(2)}\left(Y_{L}, Y_{R}\right)$ is used to compute $Y^{* *}\left(\frac{x}{t} ; Y_{L}, Y_{R}\right)$, following the same procedure as detailed above (to compute $\left.Y^{*}\right)$.

Once the exact solution $Y^{* *}\left(\frac{x}{t} ; Y_{L}, Y_{R}\right)$ of this approximate problem is obtained, the numerical flux is defined as:

$$
\phi\left(W_{L}, W_{R}\right)=F\left(W\left(Y^{* *}\left(0 ; Y_{L}, Y_{R}\right)\right)\right)
$$

The explicit form of the Finite Volume method called WFRoe will be:

$$
W_{i}^{n+1}-W_{i}^{n}+\frac{\Delta t}{\Delta x}\left(F\left(W\left(Y^{* *}\left(0 ; Y_{i}^{n}, Y_{i+1}^{n}\right)\right)\right)-F\left(W\left(Y^{* *}\left(0 ; Y_{i-1}^{n}, Y_{i}^{n}\right)\right)\right)\right)=0
$$

An important remark:

In some situations, the eigenvectors of $B(\hat{Y})$ do not depend on $\hat{Y}$. An example will be given below (Model A). In that case, the intermediate states will not depend on the choice of $\hat{Y}$, but the numerical wave speeds will nonetheless depend on $\hat{Y}$. Thus $Y^{*}\left(0 ; Y_{i-1}^{n}, Y_{i}^{n}\right)$ is different from $Y^{* *}\left(0 ; Y_{i-1}^{n}, Y_{i}^{n}\right)$. Nevertheless, a new iteration using $\hat{Y}^{(3)}\left(Y_{L}, Y_{R}\right)=Y^{* *}\left(0 ; Y_{L}, Y_{R}\right)$ would lead to the same scheme.

\section{A two-equation model to describe the motion of particles (model A)}

In a one dimensional framework, the motion of particles in a vacuum of gas may be written as the conservation of mass and momentum, using a pressure law to account for intergranular effects. Thus the model A may be written in a conservative form, using conservative variable $W={ }^{t}(\alpha, \alpha U)$, and noting $\alpha$ the void fraction of particles -which is assumed to lie in $I_{a d m}=\left[0, \alpha_{\max }\right]-$. We recall that a typical value for the upper bound is $\alpha_{\max }=0.64$. Moreover, $U$ and $Q=\alpha U$ will stand for the particle velocity and the particle momentum respectively:

$$
\begin{array}{r}
\frac{\partial \alpha}{\partial t}+\frac{\partial \alpha U}{\partial x}=0 \\
\frac{\partial \alpha U}{\partial t}+\frac{\partial \alpha U^{2}+\theta(\alpha)}{\partial x}=\frac{\partial \mu \frac{\partial U}{\partial x}}{\partial x}
\end{array}
$$


$\mu$ is a positive constant, and the fucntion $\theta(\alpha)$ is increasing, and agrees with $\theta(0)=0$ and $\lim _{\alpha->\alpha_{\max }}(\theta(\alpha))=$ $+\infty$. For various choices of functions $\theta(\alpha)$, we refer for instance to references $,{ }^{11},{ }^{12},{ }^{13},{ }^{14}$ and references therein. We will detail some specific forms afterwards when computing this system.

Property A

We set :

$$
\begin{gathered}
\eta(W)=\frac{\alpha U^{2}}{2}+H(\alpha) \\
H(\alpha)=\alpha \int_{0}^{\alpha}\left(\frac{\theta(a)}{a^{2}} d a\right) \\
F_{\eta}(W)=U(\eta(\alpha)+\theta(\alpha))
\end{gathered}
$$

The following entropy inequality holds for system $((4),(5))$ :

$$
\frac{\partial \eta(W)}{\partial t}+\frac{\partial F_{\eta}(W)}{\partial x} \leq 0
$$

The proof is rather classical and is not detailed herein. The reader is refered to, ${ }^{152}$ for instance.

Introducing $c(a)=\left((\theta)^{\prime}(a)\right)^{1 / 2}$, we may check that the convective set (or left hand side) of system $((4),(5)$ ) is hyperbolic. Eigenvalues are : $\lambda_{1}=U-c, \lambda_{2}=U+c$, and both fields are genuinely non linear, as happens for shallow-water equations. The Riemann invariants in the 1 -wave and the 2 -wave are $I^{+}=U+g(\alpha)$, and $I^{-}=U-g(\alpha)$ respectively, where $: g(\alpha)=\int_{0}^{\alpha}\left(\frac{c(a)}{a} d a\right)$.

\section{Proposition A}

The one-dimensional Riemann problem associated with the LHS of $((4),(5))$ has a unique entropy consistent solution composed of constant states separated by shocks and rarefaction waves, with no vacuum occurence, provided that the initial data $\left(\alpha_{L}, U_{L}\right),\left(\alpha_{R}, U_{R}\right)$ agrees with the following condition:

$$
U_{R}-U_{L}<g\left((\alpha)_{L}\right)+g\left((\alpha)_{R}\right)
$$

still setting : $g(a)=\int_{0}^{\alpha} \frac{c(a)}{a} d a$. Moreover, the maximum principle for the void fraction holds, which means that the solution $\alpha(x, t)$ of the Riemann problem lies in $I_{a d m}$.

Proof:

The first part of the proof is again rather classical,,${ }^{15}{ }^{2}$ The only specific point concerns the preservation of the upper bound for $\alpha(x, t)$. Actually, the void fraction increases through shocks only (from the left to the right in a 1-wave, and from the right to the left in a 2-wave). The only situation which requires attention is the one where the initial data generates a double shock wave. Otherwise, the intermediate value $\alpha_{1}$ (indexing by 1 the intermediate state in the solution of the Riemann problem ) lies between $\min \left(\alpha_{L}, \alpha_{R}\right)$ and $\max \left(\alpha_{L}, \alpha_{R}\right)$. Thus, if the two waves happen to be shock waves, we have, setting as usual $(\Delta \phi)_{l r}=\phi_{r}-\phi_{l}$ for given left and right states on each side of a discontinuity:

$$
\sigma_{1}=(\Delta \alpha U)_{L 1} /(\Delta \alpha)_{L 1}
$$

and :

$$
U_{1}=U_{L}-\left(\frac{(\Delta \alpha)_{L 1}(\Delta \theta(\alpha))_{L 1}}{\alpha_{L} \alpha_{1}}\right)^{1 / 2}=U_{L}-f_{L}\left(\alpha_{1}\right)
$$

but also :

$$
\sigma_{2}=(\Delta \alpha U)_{1 R} /(\Delta \alpha)_{1 R}
$$

and :

$$
\begin{gathered}
U_{R}=U_{1}-\left(\frac{(\Delta \alpha)_{1 R}(\Delta \theta(\alpha))_{1 R}}{\alpha_{R} \alpha_{1}}\right)^{1 / 2}=U_{1}-f_{R}\left(\alpha_{1}\right) \\
5 \text { of } 14
\end{gathered}
$$


Adding the second equation 9 to the fourth one (11) enables to eliminate the unknown velocity $U_{1}$, and then obtain the solution in terms of $\alpha_{1}$, solution of:

$$
U_{R}-U_{L}=-f_{L}\left(\alpha_{1}\right)-f_{R}\left(\alpha_{1}\right)
$$

It can be easily checked that the positive solution $\alpha_{1}$ is bounded : $\alpha_{1}<\alpha_{\max }$, owing to the crucial property : $\lim _{\alpha->\alpha_{\max }}(\theta(\alpha))=+\infty$. This completes the proof.

The previous analysis provides an interesting basic experiment. This one corresponds either to an impinging jet on a wall boundary, or equivalently to two identical jets coming one to the other in a cylindrical tube, while neglecting viscous effects. Owing to the previous analogy, we will denote it the IJWB test (Impinging Jet on a Wall Boundary). The resulting flow pattern in the central region (or the wall interface respectively) is a flow at rest with increasing granular pressure. This corresponds to a Riemann problem with initial data $W_{L}=\left(\alpha_{R},-\alpha_{R} U_{R}\right)$ and $W_{R}=\left(\alpha_{R}, \alpha_{R} U_{R}\right)$, with $U_{R}<0$. Still denoting 1 the subscript for the intermediate state, we have due to symmetry $U_{1}=0$ and the unique solution $\alpha_{1}$ in $I_{a d m}$ is given through the solution of : $\frac{[\alpha]_{1}^{R} /[\theta(\alpha)]_{1}^{R}}{\alpha_{1}}=\alpha_{R}\left(U_{R}\right)^{2}$.

\section{Remark}

In some situations, the governing equation for the total energy of particles cannot be disregarded. Hence a natural counterpart of model A, will be governed by full Euler equations with a particular form of the internal energy.

\section{A three equation hyperbolic conservative mixture model (model B)}

We now consider a medium of gas (phase 1) loaded with heavy particles with constant density $\rho_{2}$ (phase 2 ). We note $\alpha_{2}$ the void fraction of particles, and $\rho_{1}$ the density of the gas. As usual, we will define $P\left(\alpha_{2}, \rho_{1}\right)$ the mean pressure in the two phase flow, and will assume that the relative velocity is small compared with the speed of density waves. A simple though efficient way to describe the motion of the two phase mixture is to write conservation of mass of gas, mass of particles, and the mean momentum equation, that is to say:

$$
\frac{\partial W}{\partial t}+\frac{\partial F(W)}{\partial x}=\frac{\partial G^{v i s c}}{\partial x}
$$

with :

$$
W=\left(\begin{array}{c}
\left(1-\alpha_{2}\right) \rho_{1} \\
\alpha_{2} \rho_{2} \\
\tilde{\rho} U
\end{array}\right) \quad \text { and } \quad F(W)=\left(\begin{array}{c}
\left(1-\alpha_{2}\right) \rho_{1} U \\
\alpha_{2} \rho_{2} U \\
\tilde{\rho} U^{2}+P
\end{array}\right)
$$

The viscous flux contribution is provided through :

$$
G^{v i s c}=\left(0,0, \mu \frac{\partial U}{\partial x}\right)^{t}
$$

The mean pressure sums two contributions, thus : $P\left(\alpha_{2}, \rho_{1}\right)=p\left(\rho_{1}\right)+\rho_{2} \theta\left(\alpha_{2}\right)$. The mean density is the sum of partial masses:

$$
\tilde{\rho}=\left(1-\alpha_{2}\right) \rho_{1}+\alpha_{2} \rho_{2}
$$

The $\theta\left(\alpha_{2}\right)$ function is similar to the one described above in section 2. Both functions $p$ and $\theta$ are assumed to be monotone increasing wrt their argument. One also needs for conveniency to introduce celerities in isolated phases, that is:

$$
\begin{aligned}
c_{1} & =\left(\frac{\partial P\left(\alpha_{2}, \rho_{1}\right)}{\partial \rho_{1}}\right)^{1 / 2} \\
c_{2} & =\left(\frac{1}{\rho_{2}} \frac{\partial P\left(\alpha_{2}, \rho_{1}\right)}{\partial \alpha_{2}}\right)^{1 / 2}
\end{aligned}
$$

$$
6 \text { of } 14
$$


but also :

$$
c\left(\alpha_{2}, \rho_{1}\right)=\left(\frac{\left(1-\alpha_{2}\right) \alpha_{2} \rho_{2} c_{2}^{2}+\rho_{1} c_{1}^{2}}{\alpha_{1} \tilde{\rho}}\right)^{1 / 2}
$$

This model may be obtained on the basis of a two fluid model by assuming local instantaneous velocity equilibrium, and adding both momentum equations within each phase (for instance the basic model of Gidaspow, see, ${ }^{16},{ }^{17}$ or alternatively the non conservative two-fluid model of ${ }^{18}$ ).

The convective subset of system (13) is hyperbolic, since the Jacobian matrix has three distinct eigenvalues $: \lambda_{1}=U-c, \lambda_{2}=U, \lambda_{3}=U+c$. Both the 1-wave and the 3-wave are genuinely non linear, and the 2-wave is linearly degenerated. The Riemann invariants in the 1 -wave and the 3 -wave are: $\left(s=\frac{\left(1-\alpha_{2}\right) \rho_{1}}{\alpha_{2} \rho_{2}}, I^{+}=\right.$ $\left.U+\psi\left(s, \rho_{1}\right)\right)$, and: $\left(s, I^{-}=U-\psi\left(s, \rho_{1}\right)\right)$ respectively, denoting:

$$
\psi(s, \rho)=\int_{0}^{\rho}\left(\frac{c\left(s, \rho_{1}\right)}{\rho_{1}\left(1+\frac{\rho_{1}}{\rho_{2} s}\right)} d \rho_{1}\right)
$$

The Riemann invariants in the 2-wave are: $(U, P)$.

Property B

If we set :

$$
\begin{gathered}
\eta(W)=\frac{\tilde{\rho} U^{2}}{2}+H\left(\alpha_{2}, \rho_{1}\right) \\
H\left(\alpha_{2}, \rho_{1}\right)=\alpha_{1} \rho_{1} \int_{0}^{\rho_{1}}\left(\frac{p(a)}{a^{2}} d a\right)+\alpha_{2} \rho_{2} \int_{0}^{\alpha_{2}}\left(\frac{\theta(\alpha)}{\alpha^{2}} d \alpha\right) \\
F_{\eta}(W)=U\left(\eta(W)+P\left(\alpha_{2}, \rho_{1}\right)\right)
\end{gathered}
$$

The following entropy inequality holds for system (13):

$$
\frac{\partial \eta(W)}{\partial t}+\frac{\partial F_{\eta}(W)}{\partial x} \leq 0
$$

\section{Proposition B}

The one-dimensional Riemann problem associated with the LHS of (13) has a unique entropy consistent solution composed of constant states separated by shocks, contact discontinuities and rarefaction waves, with no vacuum occurence, provided that the initial data $\left(\left(\alpha_{2}\right)_{L},\left(\rho_{1}\right)_{L}, U_{L}\right),\left(\left(\alpha_{2}\right)_{R},\left(\rho_{1}\right)_{R}, U_{R}\right)$ complies with :

$$
U_{R}-U_{L}<\psi\left(s_{L},\left(\rho_{1}\right)_{L}\right)+\psi\left(s_{R},\left(\rho_{1}\right)_{R}\right)
$$

The maximum principle for the void fraction holds, since the solution $\alpha_{2}(x, t)$ of the Riemann problem lies in $I_{a d m}$.

The reader is refered to ${ }^{19}$ for proof.

\section{A Finite Volume scheme to compute model A}

We note here again:

$$
g(\alpha)=\int_{0}^{\alpha} \frac{c(a)}{a} d a
$$

still using $c(a)=\left(\frac{\partial \theta}{\partial a}\right)^{1 / 2}$. The LHS of system $((4),(5))$ may be rewritten in terms of the non conservative variable $Y(W)={ }^{t}(g, u)$ in a symmetrized form:

$$
\frac{\partial Y}{\partial t}+C(Y) \frac{\partial Y}{\partial x}=0
$$

$$
7 \text { of } 14
$$


noting:

$$
C(Y)=\left(\begin{array}{ll}
u & c \\
c & u
\end{array}\right)
$$

The matrix $R(Y)$ of right eigenvectors associated with $\lambda_{1}=u-c$ and $\lambda_{2}=u+c$ simply is :

$$
R(Y)=\left(\begin{array}{rr}
1 & 1 \\
-1 & 1
\end{array}\right)
$$

We turn now to the associated linearised problem:

$$
\frac{\partial Y}{\partial t}+C\left(\hat{Y}_{L R}\right) \frac{\partial Y}{\partial x}=0
$$

We still note for any quantity $\phi:(\Delta \phi)_{L R}=\phi_{R}-\phi_{L}$ and $(\bar{\phi})_{L R}=\left(\phi_{R}+\phi_{L}\right) / 2$. The computation of the intermediate state in the linearised solver at each interface between two cells labelled $L, R$ is recalled in section II. Whatever the value of $\hat{Y}$ is, the intermediate states are:

$$
g_{1}=\bar{g}_{L R}-\frac{(\Delta U)_{L R}}{2} \quad \text { and } \quad U_{1}=\bar{U}_{L R}-\frac{(\Delta g)_{L R}}{2}
$$

\section{A. Main properties}

Property $C$ :

(a)-Vacuum arises in the intermediate state of linearized Godunov solver if and only if initial data makes vacuum occur in the exact solution of the Riemann problem associated with the non linear set of equations:

$$
u_{R}-u_{L}<g_{L}+g_{R}
$$

(b) - The upper bound of the intermediate state in the solution of the linearised Riemann problem is preserved if the condition holds on $\theta(\alpha))$ :

$$
\int_{0}^{\alpha_{\max }}\left(\frac{c(a)}{a} d a\right)=+\infty
$$

Proof:

(a) Owing to the expression of the intermediate $g_{1}$ given above, we immediately check that $g_{1}>o$ is equivalent to $u_{R}-u_{L}<g_{L}+g_{R}$, which is precisely the exact condition of occurence of vacuum. Since the function $g(\alpha)$ is increasing, and $g(0)=0$, this guarantees positive values of the intermediate state $\alpha_{1}$ such that $g\left(\alpha_{1}\right)=g_{1}$.

(b) The condition $\int_{0}^{\alpha_{\max }}\left(\frac{c(a)}{a} d a\right)=+\infty$ obviously implies that the solution $\alpha_{1}$ of equation $g\left(\alpha_{1}\right)=g_{1}$ will be smaller than $\alpha_{\max }$. This completes the proof.

The second condition is clearly related to the choice of the approximate Godunov scheme, since the latter no longer distinguishes shock waves from rarefaction waves, unlike Godunov scheme.

\section{B. The particular case of the IJWB}

We focus thus on the initial data $W_{L}=\left(\alpha_{L}, \alpha_{L} U_{L}\right)$ and $W_{R}=\left(\alpha_{L},-\alpha_{L} U_{L}\right)$, with $U_{L}>0$, and evenmore assume that $\alpha_{R}<<\alpha_{\max }$. We first note that due to the structure of $R(Y)$, intermediate states $g_{1}$, $u_{1}$ do not depend on the average $\hat{Y}$ (see above). A straightforward computation shows that the CFL condition for VFRoe-ncv and WFRoe requires evaluations of $\left.\lambda_{1}^{(1)}=\bar{U}_{L R}-\bar{c}_{L R}=-c_{(} \alpha\left(g_{L}\right)\right)$ and $\lambda_{1}^{(2)}=U(Y *)-c(Y *)=$ $-c(\alpha *)=-c\left(\alpha\left(g_{L}\left(1+u_{L} / g_{L}\right)\right)\right.$ respectively. The ratio of the modulus of both may be set equal to any 
positive constant A. Thus, we can imagine that the time step based on the basic VFRoe-ncv scheme will be totally diferent from the one obtained with WFRoe.

\section{A Finite Volume scheme to compute model B}

We may write in a different way the three-equation model B. Still defining $s=\frac{\alpha_{1} \rho_{1}}{\alpha_{2} \rho_{2}}$, and restricting to regular solutions, the following holds:

$$
\frac{\partial Y}{\partial t}+C(Y) \frac{\partial Y}{\partial x}=0
$$

with:

$$
Y(W)={ }^{t}(s, U, P)
$$

and:

$$
C(Y)=\left(\begin{array}{ccc}
u & 0 & 0 \\
0 & u & \frac{1}{\tilde{\rho}} \\
0 & \tilde{\rho} c^{2} & u
\end{array}\right)
$$

Right eigenvectors of $C(Y)$ are:

$$
r_{1}(Y)=\left(\begin{array}{c}
0 \\
1 \\
-\tilde{\rho} c
\end{array}\right), r_{2}(Y)=\left(\begin{array}{c}
1 \\
0 \\
0
\end{array}\right), r_{3}(Y)=\left(\begin{array}{c}
0 \\
1 \\
\tilde{\rho} c
\end{array}\right) .
$$

Rather than computing the exact solution of the Riemann problem, we consider the linearised problem:

$$
\frac{\partial Y}{\partial t}+C\left(\hat{Y}_{L R}\right) \frac{\partial Y}{\partial x}=0
$$

Eigenvalues are: $\tilde{\lambda}_{1}=U(\hat{Y})-c(\hat{Y}), \tilde{\lambda}_{2}=U(\hat{Y}), \tilde{\lambda}_{3}=U(\hat{Y})+c(\hat{Y})$. Coefficients arising in the decomposition of $Y_{R}-Y_{L}$ on the basis of right eigenvectors of $C(\hat{Y})$ enable to compute the two intermediate states occuring in the solution of the linearised Riemann problem. Thus we have:

$$
\begin{aligned}
& Y_{1}=\left(\begin{array}{c}
s_{1} \\
U_{1} \\
P_{1}
\end{array}\right) \quad \text { and } Y_{2}=\left(\begin{array}{c}
s_{2} \\
U_{2} \\
P_{2}
\end{array}\right) . \\
& s_{1}=s_{L} \\
& s_{2}=s_{R} \\
& U_{1}=U_{2}=\bar{U}_{L R}-\frac{1}{2 \tilde{\rho}(\hat{Y}) c(\hat{Y})}(\Delta P)_{L R} \\
& P_{1}=P_{2}=\bar{P}_{L R}-\frac{\tilde{\rho}(\hat{Y}) c(\hat{Y})}{2}(\Delta U)_{L R}
\end{aligned}
$$

Property D:

The intermediate value $P_{1}=P_{2}$ is positive provided that the initial conditions of the Riemann problem agree with condition:

$$
(\Delta U)_{L R}<\frac{2 \bar{P}_{L R}}{\tilde{\rho}\left(\hat{Y_{L R}}\right) c\left(\hat{\left.Y_{L R}\right)}\right.}
$$

$$
9 \text { of } 14
$$


in that case, the intermediate values of density $\rho_{1}$ and $\alpha_{2}$ remain positive.

Proof:

the first part of the proof is obvious due to the expression of $P_{1}=P_{2}=\tilde{P}_{L R}$. We may now assume that $\tilde{P}_{L R}$ is positive. The first intermediate states of density $\left(\rho_{1}\right)_{1}$ and $\left(\alpha_{2}\right)_{1}$ are solutions to:

$$
P\left(\left(\alpha_{2}\right)_{1},\left(\rho_{1}\right)_{1}\right)=\tilde{P}_{L R} \quad \text { and } \quad \frac{\left(1-\left(\alpha_{2}\right)_{1}\right)\left(\rho_{1}\right)_{1}}{\left(\alpha_{2}\right)_{1} \rho_{2}}=s_{L}
$$

eliminating $\left(\rho_{1}\right)_{1}=s_{L} \frac{\left(\alpha_{2}\right)_{1} \rho_{2}}{\left(1-\left(\alpha_{2}\right)_{1}\right)}$ and putting in the former equation provides $\left(\alpha_{2}\right)_{1}$ solution of equation: $h\left(\left(\alpha_{2}\right)_{1}\right)=\tilde{P}$ where $h(a)=P\left(a, \frac{s_{L} \rho_{2} a}{1-a}\right)$. The function $h(a)$ is monotone increasing since $h^{\prime}(a)=\rho_{2}\left(c_{2}^{2}+\right.$ $\left.c_{1}^{2} \frac{s_{L}}{(1-a) 2}\right)>0$. Moreover, $h(0)=P\left((0,0)=0\right.$ and $\lim _{a->\alpha_{m a x}^{-}} h(a)=+\infty$. Thus the previous non linear scalar equation has only one solution $\left(\alpha_{2}\right)_{1}$ in the admissible range $I_{a d m}$. Therefore, the intermediate density $\left(\rho_{1}\right)_{1}=s_{L} \frac{\left(\alpha_{2}\right)_{1} \rho_{2}}{\left(1-\left(\alpha_{2}\right)_{1}\right)}$ is positive, which completes the proof, since a similar result holds for both $\left(\rho_{1}\right)_{2}$ and $\left(\alpha_{2}\right)_{2}$.

\section{A few numerical results}

The reader is refered to some enclosed results which depict the behaviour of WFRoe scheme when computing the IJWB case, setting $\alpha_{L}=0.4$ and $U_{L}=10$ (first series) $\alpha_{L}=0.4$ and $U_{L}=20$ (second series respectively). The CFL constant has been set to 0.4. The form of the intergranular pressure implies here that : $c(\alpha)=\frac{\alpha_{\max }}{\alpha_{\max }-\alpha}$. The exact intermediate state of the void fraction in the first example is indeed very close to $\alpha_{\max }=0.64$ (approximately 0.63708); in the second series $(C F L=0.4,8000$ cells, 40000 time steps), an approximation of the intermediate state is: 0.63906 .

One may notice that close to the shock wave(s), the velocity profile is not perfectly monotone. We recall that the exact Godunov scheme generates a similar behaviour. We emphasize that the mesh refinement confirms that the scheme remains stable.

The last figure shows the behaviour of WFRoe when using initial condition $W_{L}=\left(\alpha_{L}, \alpha_{L} U_{L}\right)$ and $W_{R}=\left(\alpha_{L},-\alpha_{L} U_{L}\right)$, setting $\alpha_{L}=0.4$ and $U_{L}=-100$, which results in a strong double rarefaction wave with a vacuum of particles. The time step agrees with the CFL condition $C F L=0.4$, and the mesh contains 200 cells.

\section{Conclusion}

On the basis on several numerical experiments, the approximate Godunov scheme WFRoe seems to be an interesting candidate for numerical industrial situations, when one cannot afford solving the exact Godunov scheme on each cell interface, which is the case in practice in our framework. It more or less enables to anticipate stiff time variations, and thus enables to control the time step on the basis of interface values. We wish to emphasize here that WFRoe scheme does not increase the accuracy of VFRoe scheme, but only affects the nonlinear stability of the whole cell scheme. Of course, this method may also be used to compute systems arising in references, ${ }^{20},{ }^{21},{ }^{17},{ }^{22}$ or any others involving acoustic speeds which depend much on one argument. It also seems to be a promosing way to improve Finite Volume schemes dedicated to the approximation of solutions of the shallow water equations with bottom gradients. The main reason in that case is that the local velocity (defined as the ratio of the mass flow rate over the water height) is much sensistive in near-vacuum areas. 


\section{Figures}

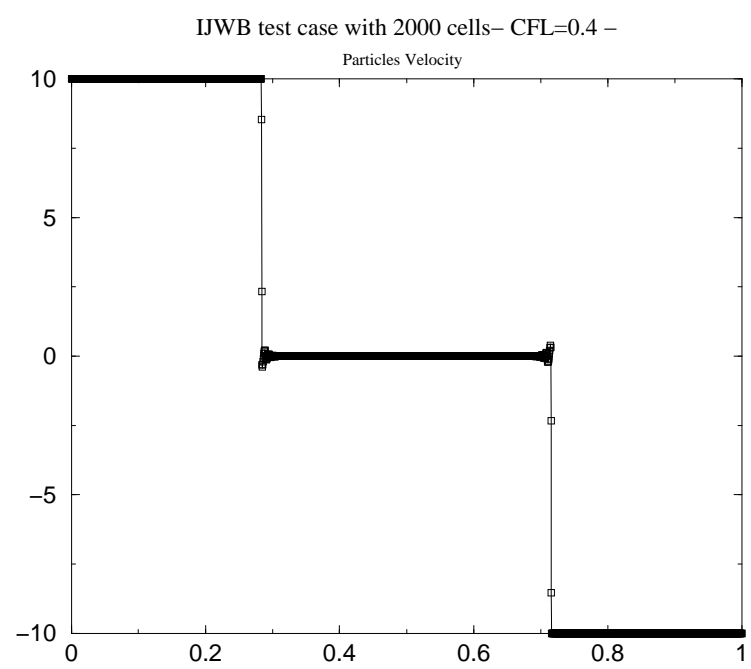

Figure 1. Velocity in impinging jets (two-equation model A). The wall is located at $\mathrm{x}=0.5$

\section{References}

${ }^{1}$ S.K. Godunov, A difference method for numerical calculation of discontinuous equations of hydrodynamics, Mat. Sb., 1959, pp. 271-300. In Russian.

${ }^{2}$ E. Godlewski ANd P.A. Raviart, Numerical approximation of hyperbolic systems of conservation laws, Springer Verlag, 1996.

${ }^{3}$ B. Einfeldt, C.D. Munz, P.L. Roe And B. Sjögreen, On Godunov-type methods near low densities, J. Comp. Phys., 1991, vol. 92-2, pp. 273-295.

${ }^{4}$ M.S. Liou, A sequel to AUSM : AUSM+, J. Comp. Phys., 1996, vol.129, pp.364-382.

${ }^{5}$ T. Buffard, T. Gallouët And J.M. HÉrard, A sequel to a rough Godunov scheme. Application to real gas flows, Computers and Fluids, 2000, vol. 29-7, pp. 813-847.

${ }^{6}$ T. Gallouët, J.M. HÉrard and N. Seguin, Some recent Finite Volume schemes to compute Euler equations using real gas EOS, Int. J. for Num. Meth. in Fluids, 2002, vol. 39(12), pp. 1073-1138.

${ }^{7}$ T. Galloü̈t, J.M. HÉrard and N. Seguin, Some approximate Godunov schemes to compute shallow water equations with topography, Computers and Fluids, 2003, vol. 32(4), pp.479-513.

${ }^{8}$ T. Gallouët, J.M. HÉrard and N. SEguin, A hybrid scheme to compute contact discontinuities in one dimensional Euler systems, Math. Mod. and Num. Analysis, 2003, vol. 14(5), pp.663-700.

${ }^{9}$ T. Gallouët, J.M. Hérard and N. Seguin, On the use of some symetrizing variables to deal with vacuum, Calcolo,2003, vol. 40(3), pp.163-194.

${ }^{10}$ R.J. LeVeque, Numerical Methods for Conservation Laws, Birkhäuser-Verlag, Basel, 1990.

${ }^{11}$ J.T. Jenkins and S.B. Savage, A theory for the rapid flow of identical smooth nearly elastic particles, Journal of Fluid Mechanics, 1983, vol. 130, pp. 187-202.

${ }^{12}$ C.K.K. Lun, S.B. Savage, D.J. Jeffrey and N. Chepurniy, Kinetic theories for granular flows: inelastic particles in Couette flow and slightly inelastic particles in a general flowfield, Journal of Fluid Mechanics, 1984, vol. 140, pp. $223-256$.

${ }^{13}$ C.K.K. Lun And S.B. SAvage, The effect of an impact dependent coefficient of restitution on stresses developped by sheared granular materials, Acta Mechanica, 1986, vol. 63.

${ }^{14}$ A. Goldshtein, M. Shapiro And C. Gutfinger, Mechanics of colisional motion of granular materials. Part IV: self similar shock wave propagation, Journal of Fluid Mechanics, 1996, vol. 316, pp. 29-51.

${ }^{15}$ J. SMOLLER, Shock waves and reaction diffusion equations, Springer Verlag, 1983.

${ }^{16}$ D. Gidaspow, Multiphase flow and fluidization, Academic Press, New York, 1994.

11 of 14

American Institute of Aeronautics and Astronautics 


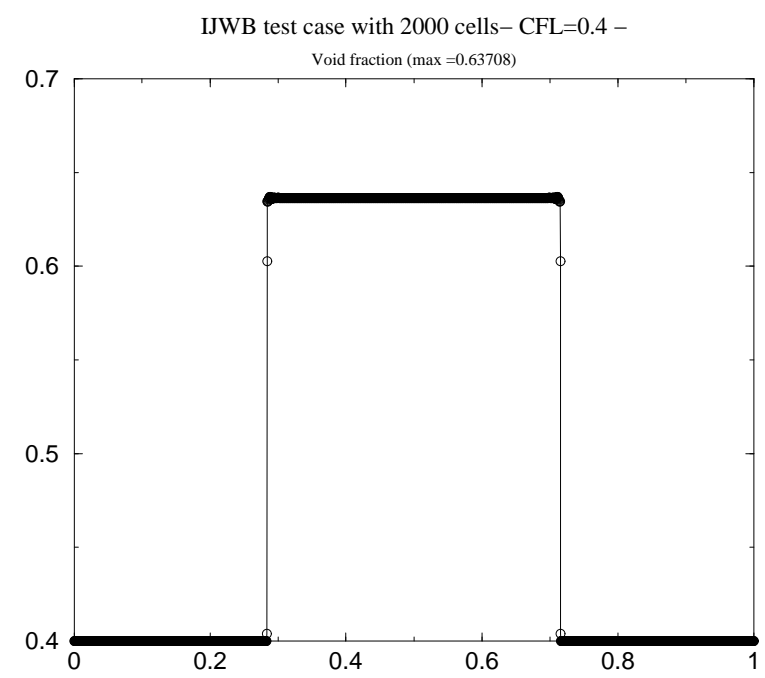

Figure 2. Void fraction in impinging jets (two-equation model A). The wall is located at $\mathrm{x}=0.5$

${ }^{17}$ D. Mao, J.R. Edwards, A.K. Kuznetsov and R.K. SRivastava, Development of low diffusion flux splitting methods for dense gas-solid flows, J. Comp. Phys., 2003, vol.185, pp.100-119.

${ }^{18}$ L. Combe And J.M. HÉrard, Finite Volume algorithm to compute dense compressiblw gas solid flows, AIAA Journal, 1999, vol. 37-3, pp. 337-345.

${ }^{19}$ L. COMbe And J.M. HÉrard, Principe du maximum pour un modèle diphasique gaz solide à trois équations,1996, EDF-DER report $H E-41 / 96 / 045 / A$.

${ }^{20}$ M.R. BAer AND J.W. Nunziato, A two phase mixture theory for the deflagration to detonation (DDT) transition in reactive granular materials, Int. J. Multiphase Flow, 1986, vol. 12-6, pp. 861-889

${ }^{21} \mathrm{~S}$. BoIvin, F. CAYré AND J.M. HÉRARD, Un schéma Volumes Finis pour la simulation d'écoulements diphasiques à deux phases incompressibles sur maillage triangulaire, Revue Européenne des Eléments Finis, 2001, vol. 10-5, pp. 539-574.

${ }^{22} \mathrm{X}$. Rogue, Expériences et simulation numérique d'écoulements diphasiques en tube à choc, PhD thesis, Université de Provence, Marseille, France, 1998. 


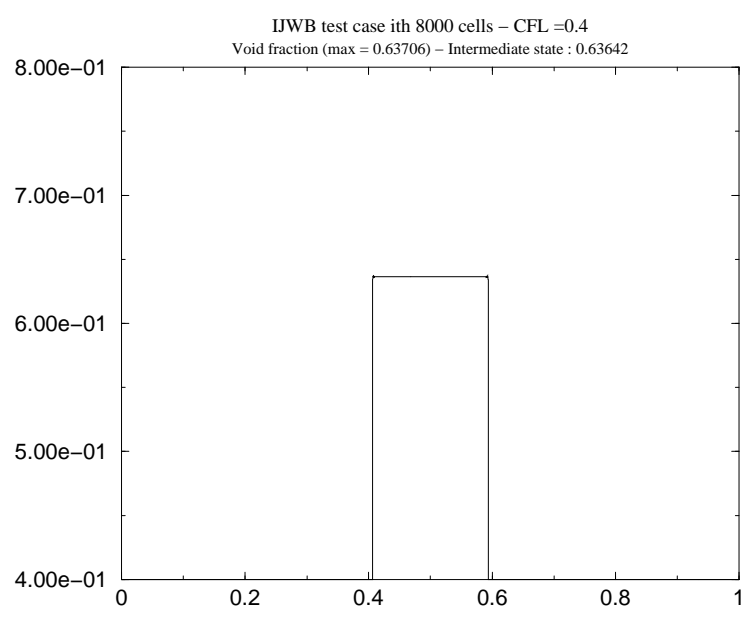

Figure 3. Void fraction in impinging jets (two-equation model A)- Refined mesh. The wall is located at $\mathrm{x}=0.5$

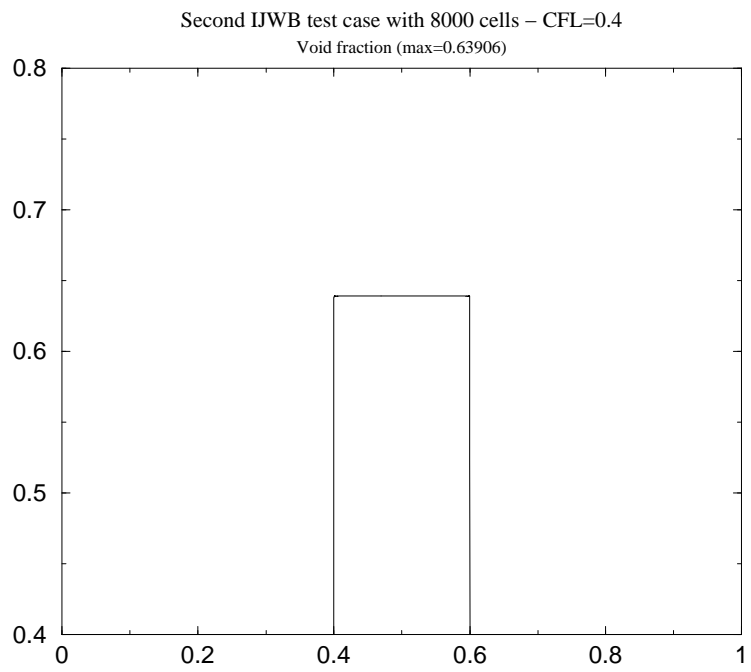

Figure 4. Void fraction in impinging jets (two-equation model A)- Refined mesh (test 2) . The wall is located at $\mathrm{x}=0.5$ 


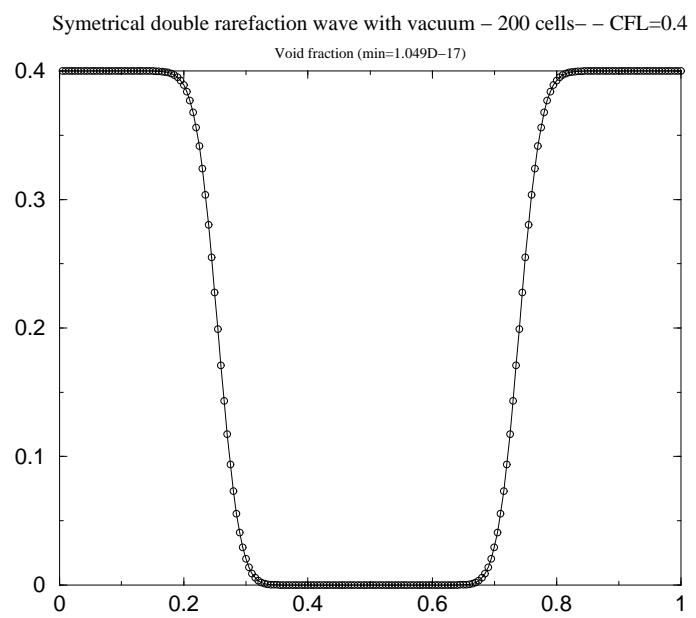

Figure 5. Void fraction in a double rarefaction wave (two-equation model $\mathrm{A}$ ). The wall is located at $\mathrm{x}=0.5$ 